\title{
Retrospective Study of Diagnostic Accuracy of Computed Tomography-Based Staging of Wilms' Tumor in the Era of Multidetector Computed Tomography
}

\section{Garima Pathak ${ }^{1}$ Seema Kembhavi ${ }^{2}$ Palak Popat ${ }^{2} \quad$ Sajid Qureshi ${ }^{3} \quad$ Mukta Ramadwar $^{4}$ Tushar Vora ${ }^{5}$}

${ }^{1}$ Department of Radiology, Tata Memorial Center, Mumbai, Maharashtra, India

2Department of Radiology, Tata Memorial Center, Mumbai, Maharashtra, India

${ }^{3}$ Department of Pediatric Surgical Oncology, Tata Memorial Center, Mumbai, Maharashtra, India

${ }^{4}$ Department of Pathology, Tata Memorial Center, Mumbai, Maharashtra, India

${ }^{5}$ Department of Pediatric Oncology, Tata Memorial Center, Mumbai, Maharashtra, India

J Gastrointestinal Abdominal Radiol ISGAR 2019;2:33-38
Address for correspondence Seema Kembhavi, DNB, Department of Radiology, Tata Memorial Center, Mumbai, Maharashtra, India (e-mail: Seema.kembhavi@gmail.com).

\section{Abstract}

Keywords

- CT staging of Wilms' tumor

- diagnostic accuracy of Wilms' CT

- interobserver agreement
Objectives To evaluate the diagnostic accuracy of multidetector computed tomography (MDCT)-based staging of Wilms' tumor (WT) in comparison with surgicalpathological staging (reference standard) and to assess the interobserver agreement in predicting CT stage for WT.

Method Retrospective audit of 50 consecutive cases of WT meeting our eligibility criteria was performed, and an electronic database of CT scan findings, intraoperative findings, and final histopathology staging were created. Two radiologists blinded to surgical and histologic findings reviewed the CT scans, using multiplanar reformations to assess various parameters pertaining to tumor extent and assign a possible stage, which was then compared with the final surgical-pathological stage. Interobserver agreement was assessed using $\mathrm{k}$-coefficient.

Results CT scan correctly staged 31 (62\%) of 50 WT cases (both observers' consensus data, all stages combined). CT accuracy was $37.5 \%$ in stage I disease, $66 \%$ in stage II disease, and $75 \%$ in stage III. There was substantial interobserver agreement (in $80 \%$ of the cases) in assigning the stage. Subset analysis showed a $100 \%$ positive predictive value (PPV) in detecting renal vein thrombosis. The negative predictive value (NPV) for ureteric involvement was 91 to $93 \%$, and that for retroperitoneal adenopathy, it was 93 to $97 \%$.

Conclusion The accuracy of CT in staging WT is dependent on the disease stage. While it overestimates the extent of the disease in early stage, it predicts the advanced stage with very good accuracy and can be used to avoid upfront surgeries in locally advanced WT. 


\section{Introduction}

Wilms' tumor (WT), the most common pediatric renal tumor, is the model of exemplary success of multimodality management of cancers with a survival rate of almost $96 \%{ }^{1}$ Conventionally surgical-pathological staging is used to decide the management; however, with the advent of multidetector computed tomography (MDCT), imaging-based prediction of staging, preoperatively, has become increasingly feasible., ${ }^{2,3}$ Though there may not be any direct therapeutic implications of predicting the stage, there is a potential of avoiding difficult surgical exercise if the diagnostic accuracy of CT staging is good. ${ }^{4}$ The existing literature states a poor correlation between radiologic and surgical-pathological staging, with accuracy reported as low as $38 \% .^{5}$ These studies are relatively old and may not have explored the potential of multiplanar reformation that can be obtained on an MDCT.

In our retrospective study, we have evaluated the diagnostic accuracy of MDCT staging of WT in comparison with surgical-pathological staging (reference standard). We also performed reliability testing using interobserver agreement in predicting CT stage through blind review of CT scans by two experienced radiologists.

\section{Materials and Methods}

\section{Study Population and Recruitment}

This study was approved by the institutional review board (IRB) and was conducted in a single institution (tertiary cancer care center). This was a retrospective study design. The inclusion criteria were histopathologic proof of WT, availability of MDCT images on PACS (Picture Archival and Communication System), and surgery and histopathology done in the institution. The exclusion criteria were non-Wilms' renal tumors and bilateral tumors. Serial 50 WT cases diagnosed between January 2013 and December 2015 and meeting our eligibility criteria were included in this study.

\section{Study Procedure}

Electronic database of CT findings, relevant intraoperative findings, and histopathology were created. Two radiologists blinded to surgical and histologic findings independently reviewed the CT scans on workstations, using "live" multiplanar reformation. The radiologists had 10 and 5 years of experience in radiology (called as observers $A$ and $\mathrm{B}$, respectively). All the $\mathrm{CT}$ scans were performed on a 16-channel MDCT machine, either Lightspeed 16 (General Electric, Milwaukee, Wisconsin, United States) or Somatom emotion (Seimens Medical Solutions, Pennsylvania, United States). Each child underwent a contrast-enhanced CT scan after an intravenous injection of $1.5 \mathrm{~mL} / \mathrm{kg}$ of nonionic iodinated contrast media.

The extent of involvement in the kidney, size of the mass, renal vein thrombosis, if present, its extent into the inferior vena cava (IVC), renal sinus involvement, pelvicalyceal system involvement, ureteric involvement, any evidence or suspicion of extracapsular spread, and adenopathy were recorded. Each radiologist assigned a CT stage independently.
This information was used for interobserver concordance assessment. For the final staging, consensus data between two radiologists were also recorded, and this was compared with surgical-pathological staging (reference standard).

For the patients who had received preoperative chemotherapy, the post-chemotherapy scans were evaluated and compared with reference standard. For the purpose of this study, in patients with metastatic disease, only local staging was compared as all patients underwent surgery as per their treatment protocol. Similarly, if patients had stage III disease purely by virtue of spillage alone, it was ignored and local extent was taken into consideration, as this study aims to evaluate the accuracy of CT scan is assessing the local extent of the disease.

\section{Statistical Analysis}

Descriptive statistics of the various morphologic features of WT on imaging were assessed. Diagnostic accuracy of radiologic staging was assessed in terms of sensitivity, specificity, positive predictive value (PPV) and negative predictive value (NPV). $\mathrm{K}$-Coefficient value was calculated for measuring the degree of agreement between the two observers.

\section{Results}

This study population showed an equal distribution between the sexes and an age range from 1 to 11 years (average age of 4.3 years) (-Table 1 ). The tumor was slightly more common on the left side with three patients having horseshoe kidney. Two patients underwent upfront surgery whereas 48 received four cycles of chemotherapy. Only one patient (with otherwise stage I disease) had intraoperative spillage, and this is not considered as stage III disease for the purpose of this study.

CT scan correctly staged the disease in $31(62 \%)$ out of 50 patients (-Table 2). CT scan understaged the disease in $2(4 \%)$ cases, whereas it led to overstaging in $17(34 \%)$ cases. There was substantial agreement between the two observers in assigning the stage in $80 \%$ of the cases leading to a k-coefficient of 0.63 (-Table 3 ).

In the final staging ( - Table 4), three out of eight patients with stage I disease had their disease staged correctly. Four

Table 1 Demographics/patients profile

\begin{tabular}{|l|l|}
\hline Study population & 50 \\
\hline \multirow{3}{*}{ Sex distribution } & Male: 25 \\
\cline { 2 - 2 } & Female: 25 \\
\hline \multirow{2}{*}{ Age distribution } & Range: $1-11 \mathrm{y}$ \\
\cline { 2 - 2 } & Average: $4.3 \mathrm{y}$ \\
\hline \multirow{2}{*}{ Laterality } & Right: 22 \\
\cline { 2 - 2 } & Left: 25 \\
\cline { 2 - 2 } & Horseshoe: 3 \\
\hline \multirow{2}{*}{ ize (in cc) } & Smallest: $12 \mathrm{cc}$ \\
\cline { 2 - 2 } & Largest: $1,800 \mathrm{cc}$ \\
\hline Average volume & $241 \mathrm{cc}$ \\
\hline
\end{tabular}


Table 2 CT stage versus surgical-pathological stage

\begin{tabular}{|l|l|l|l|l|l|}
\hline \multicolumn{2}{|c|}{} & \multicolumn{3}{|c|}{ Reference standard } & Total \\
\cline { 3 - 6 } \multicolumn{2}{|c|}{} & I & II & III & \\
\hline \multirow{2}{*}{$\begin{array}{l}\text { CT } \\
\text { scan }\end{array}$} & I & 3 & 1 & 0 & 4 \\
\cline { 2 - 6 } & II & 4 & 25 & 1 & 30 \\
\cline { 2 - 6 } & III & 1 & 12 & 3 & 16 \\
\hline \multicolumn{1}{|c|}{ Total } & 8 & 38 & 4 & 50 \\
\hline
\end{tabular}

Abbreviation: CT, computed tomography.

Table 3 Cross-tabulation for CT staging done by two observers

\begin{tabular}{|c|c|c|c|c|c|}
\hline & \multicolumn{3}{|c|}{ Observer B } & \multirow[t]{2}{*}{ Total } \\
\hline & & I & II & III & \\
\hline \multirow[t]{3}{*}{ Observer $\mathrm{A}$} & I & 4 & 0 & 1 & 5 \\
\hline & II & 0 & 25 & 4 & 29 \\
\hline & III & 0 & 5 & 11 & 16 \\
\hline Total & & 4 & 30 & 16 & 50 \\
\hline
\end{tabular}

Abbreviation: CT, computed tomography.

Table 4 Final staging accuracy of CT scan

\begin{tabular}{|l|l|l|l|l|}
\hline Stage & Sensitivity (\%) & Specificity (\%) & $\begin{array}{l}\text { PPV } \\
\text { (\%) }\end{array}$ & NPV (\%) \\
\hline I & 37.5 & 97.6 & 75 & 89 \\
\hline II & 65.7 & 58.3 & 83.3 & 35 \\
\hline III & 75 & 71.7 & 18.7 & 97 \\
\hline
\end{tabular}

Abbreviations: CT, computed tomography; NPV, negative predictive value; PPV, positive predictive value.

of the stage I patients were assigned stage II by CT, two on the basis of suspected capsular involvement and one on the basis of suspected sinus involvement. One was assigned stage III based on radiologically suspicious nodes. Thus, CT accurately staged only $37.5 \%$ of stage I cases while overstaged $62.5 \%$

Twenty-five (65.7\%) out of 38 patients with stage II disease had their disease staged correctly. Overstaging occurred in 12 (31.5\%) out of 38 patients; 6 were on the basis of suspected extracapsular spread and 5 for suspected nodal involvement whereas one case was overstaged on the basis of both being wrongly suspected. Understaging occurred in $1(2.6 \%)$ out of 38 patients stage II cases due to radiologically occult involvement of pelvicalyceal system.
Three (75\%) out of four patients with stage III disease were identified correctly based on either peritoneal involvement $(n=1)$ or adenopathy $(n=3)$. One $(25 \%)$ patient with stage III disease was assigned stage II on CT and therefore understaged due to radiologically occult retroperitoneal nodal involvement.

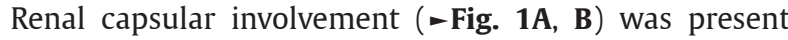
in 19 out of 50 pathology specimens. (Even though capsular involvement was present in 19 specimens, none of the resected specimens showed positive resection margin as tumor is excised along with perinephric fat and Gerota's fascia.) Observer A correctly identified renal capsular involvement in 17 cases and ruled it out in 24 cases, giving the sensitivity and specificity of $89 \%$ and $77 \%$, respectively (-Table 5). Observer B was found to be correct in identifying capsular involvement in 15 cases and ruling out in 18 cases, giving sensitivity and specificity of $79 \%$ and $58 \%$, respectively (-Table 5). The PPVs were 54\% and 71\% for observers A and B, respectively, whereas the NPVs were $82 \%$ and $92 \%$, respectively ( - Table $\mathbf{6}$ ). There was moderate strength of agreement between the two observers with $\mathrm{K}$-value $(\mathrm{K})$ being 0.44 .

Renal vein involvement ( $\boldsymbol{- F i g . ~ 2 A , ~ B ) ~ w a s ~ i d e n t i f i e d ~ i n ~}$ 7 (14\%) out of 50 cases. Sensitivity, specificity, PPV, and NPV of CT scan were $100 \%$ for both the observers in detection of renal vein thrombosis ( - Tables $\mathbf{5}, \mathbf{6}$ ). One patient had thrombosis limited to the main renal vein, two patients showed thrombi extending into the infrahepatic IVC, and three patients had thrombi extending into the retrohepatic IVC. In one patient, the tumor thrombus was stopping short of the atrium. All these extensions were correctly detected on CT.
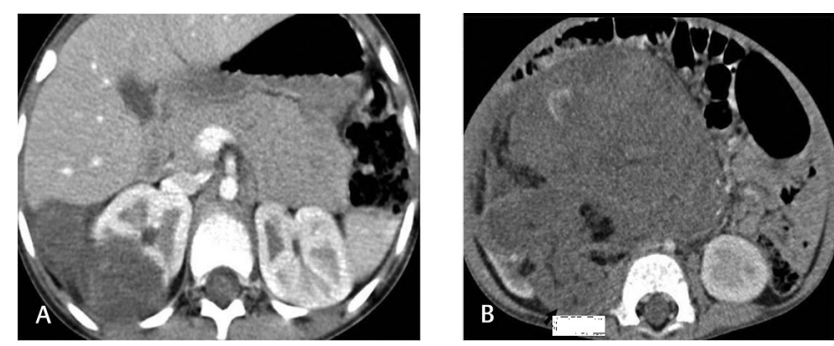

Fig. 1 (A) Axial contrast-enhanced CT image shows tumor arising from the upper pole infiltrating into perirenal soft tissue with extracapsular extension. (B) Axial contrast-enhanced CT image shows tumor arising from right kidney extending into the anterior and posterior perinephric fat (black arrow).

Table 5 Extent of disease: sensitivity and specificity for observers A and B

\begin{tabular}{|l|l|l|l|l|l|}
\hline \multirow{2}{*}{ Parameters } & \multicolumn{2}{|c|}{ Observer A } & \multicolumn{2}{c|}{ Observer B } & \multirow{2}{*}{ K } \\
\cline { 2 - 5 } & Sensitivity (\%) & Specificity (\%) & Sensitivity (\%) & Specificity (\%) & \\
\hline PCS involvement & 76 & 42 & 82 & 58 & 0.50 \\
\hline Ureteric spread & 50 & 91 & 33 & 95 & 0.69 \\
\hline Adenopathy & 25 & 83 & 75 & 83 & 0.50 \\
\hline Capsular invasion & 79 & 58 & 89 & 77 & 0.44 \\
\hline Sinus involvement & 64 & 57 & 79 & 62 & 0.67 \\
\hline Renal vein thrombus & 100 & 100 & 100 & 100 & 1.0 \\
\hline
\end{tabular}

Abbreviation: PCS, pelvicalyceal system. 
Table 6 Extent of disease: PPV and NPV for observers A and B

\begin{tabular}{|l|l|l|l|l|}
\hline \multirow{2}{*}{ Parameters } & \multicolumn{2}{|c|}{ Observer A } & \multicolumn{2}{c|}{ Observer B } \\
\cline { 2 - 5 } & PPV (\%) & NPV (\%) & PPV (\%) & NPV (\%) \\
\hline PCS involvement & 42 & 82 & 48 & 92 \\
\hline Ureteric spread & 43 & 91 & 50 & 93 \\
\hline Adenopathy & 11 & 93 & 27 & 97 \\
\hline Capsular invasion & 54 & 82 & 62 & 74 \\
\hline Sinus involvement & 67 & 52 & 100 & 100 \\
\hline Renal vein thrombosis & 100 & 100 & & 92 \\
\hline
\end{tabular}

Abbreviations: NPV, negative predictive value; PCS, pelvicalyceal system; PPV, positive predictive value.
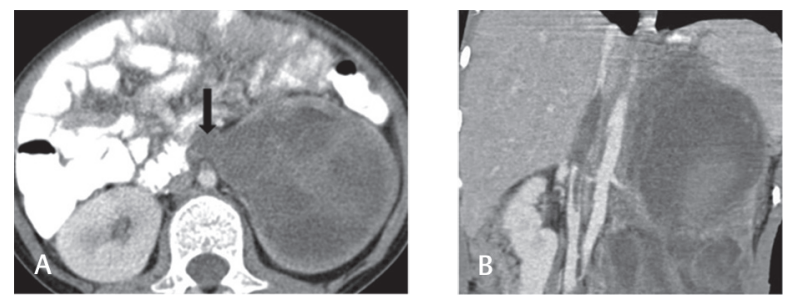

Fig. 2 (A) Axial contrast-enhanced CT image shows tumor extending into the left renal vein (black arrow). (B) Coronal reformatted image shows tumor extending into the intrahepatic segment of inferior vena cava.
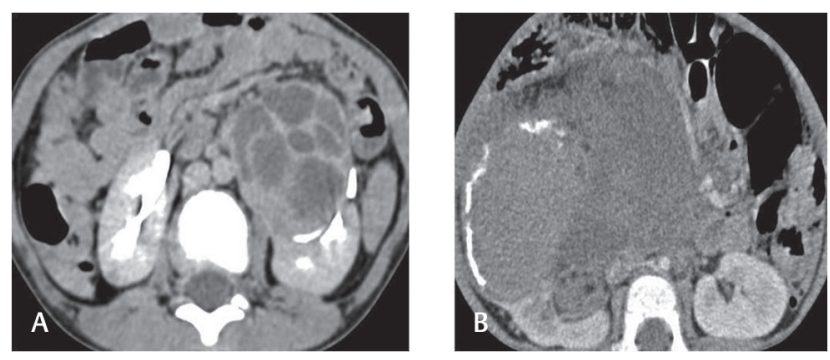

Fig. 3 (A) Three minutes delayed oblique axial CT image shows opacified pelvicalyceal system with the tumor bulging and displacing the left renal calyces; however, no definite invasion is observed. (B) Axial contrast-enhanced CT shows invasion of pelvicalyceal system and renal sinus.

Renal sinus involvement (-Fig. 3B) was present in 29 (58\%) out of 50 specimens. The sensitivity and specificity for observer A were $64 \%$ and $57 \%$, respectively, and for observer B these were 79 and 62\%, respectively (-Table 5). Good interobserver agreement was observed in assessment of renal sinus invasion on CT scan with $\mathrm{K}=0.63$. Renal pelvicalyceal system (PCS) involvement (-Fig. 3A, B) was identified in 17 (34\%) out of 50 . The sensitivity was high (76\% and $82 \%$ for observers A and B, respectively), but specificity was low for both the observers ( $42 \%$ and $58 \%$, respectively).

Ureteric involvement was present in $6(12 \%)$ out of 50 specimens. Both the observers showed high specificity but poor sensitivity in predicting ureteric involvement (-Tables 5, 6). The Interobserver agreement was very good with $\mathrm{K}=0.6$. The NPV for ureteric involvement was 91 to $93 \%$. Renal hilar and retroperitoneal adenopathy (-Fig. 4A, B) was observed in 4 (8\%) out of 50 cases, and the correlation
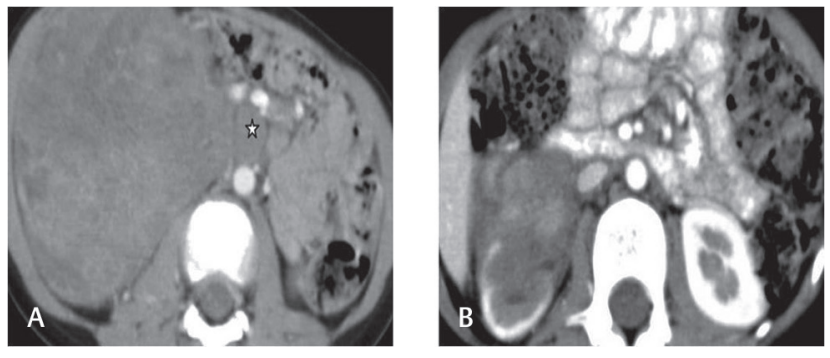

Fig. 4 (A) Axial contrast-enhanced CT image shows large tumor occupying the right kidney with preaortic lymph node (asterisk). (B) Post-chemotherapy axial CT image shows complete regression in the lymph node and significant reduction in tumor size.

was poor between the two observers. However, the NPV was high-93\% for observer A and $97 \%$ for observer B.

\section{Discussion}

The overall diagnostic accuracy of CT scan in staging of WT was found to be $62 \%$ in our study. However, as the disease stage increased, the diagnostic accuracy showed improvement: it was $37.5 \%$ in stage I disease, $66 \%$ in stage II, and $75 \%$ in stage III (-Table 4 ).

A retrospective study by Gow et al concluded that CT scan appears to have poor correlation with histologic staging. ${ }^{5}$ In their study, out of 26 WT cases, CT scan correctly staged only 10 (38\%) cases. None of the patients with stage I disease had their disease staged correctly. They were overstaged as II and III; $66 \%$ of cases with stage II disease and 60\% of stage III were staged correctly. They found that CT has difficulty for discriminating between stages I and II disease and also stages II and III disease. They also found a consistent difficulty in determining capsular involvement or nodal involvement.

Another prospective study performed by Abdelmaboud et al on 32 WT children concluded that MDCT represents a reliable diagnostic method for evaluating WT. It is accurate and sensitive in diagnosing stages I, IV, and V but is not as accurate in the diagnosis of stages II and III and has poor correlation with histopathologic data. ${ }^{6}$

A retrospective review of 179 cases by Abdelhalim et al concluded that the validity of CT staging of childhood renal tumors remains doubtful. ${ }^{7}$ The authors of this publication find that the CT is more accurate for tumors without extracapsular extension. In their study, CT overstaged $21.4 \%, 65.2 \%$, and 
$46.5 \%$ of tumors in the upfront surgery, pre-chemotherapy, and post-chemotherapy scans, respectively, and understaged $10.7 \%, 3 \%$, and $4.7 \%$, respectively.

Thus, the previously published data revealed a poor correlation between radiologic and pathologic staging.

In our study, the accuracy of CT staging is better than what has previously been reported and could be attributed to MDCT. Robust CT staging is not required to replace the well-established surgical-pathological staging systems that form an integral part of treatment protocols used across the globe. Currently, stage III disease requires a multimodal treatment: chemotherapy, surgery, and radiotherapy, whereas stage II disease is treated with chemotherapy and surgery alone. ${ }^{8}$ Moreover, chemotherapy for stages I and II comprises two drugs-vincristine and dactinomycinwhereas that for stage III also includes doxorubicin in addition to these two drugs. ${ }^{8}$

Thus, if we could use CT scan to identify extrarenal disease like infiltration of adjacent organs, adenopathy, ureteric involvement, peritoneal disease, and tumor thrombus that could potentially be stage III disease, we could avoid upfront surgery. Our study shows 97\% NPV for stage III disease, which means that if CT scan does not identify extrarenal disease, it is unlikely to be present. Identification of such disease status may not be relevant where "state of the art" cancer centers are available as the pediatric oncology team is likely to have experts in surgery, radiotherapy, and chemotherapy to offer the multimodal treatment. ${ }^{4}$ However, in resource-constrained countries or areas where radiotherapy may not be available, an accurate $\mathrm{CT}$ staging can help in triaging patients at the right time. For example, patients with probable stages I and II disease may undergo surgery followed by chemotherapy while caution would be advocated in suspected stage III disease and patients may be preferably referred to a tertiary cancer center at the right time. This may help in achieving better outcomes at smaller centers and reduce the burden on tertiary centers. In effect, CT scan staging will contribute in appropriate resource allocation.

In our study, when we did subset analysis such as predicting tumor thrombus into the IVC or retroperitoneal adenopathy, imaging was found to be useful ( - Table 7 ). For example, CT scan demonstrated the presence and even the extent of tumor thrombus in the renal vein or IVC with $100 \%$ accuracy. Prior knowledge of extent of thrombus helps in better planning the surgery. Imaging is also useful for ruling out disease in extracapsular structures; for example, in our study, NPV for ureteric involvement was 91 to $93 \%$ and that for retroperitoneal adenopathy was 93 to $97 \%$. In another retrospective review of $26 \mathrm{CT}$ scans by Silva et al, ${ }^{9} \mathrm{CT}$ tumor showed low specificity and low PPV in the detection of lymph node dissemination; however, the reported NPV was $100 \%$.

Evaluation of the renal sinus and PCS is poor probably because of large size of these tumors. Both these can get compressed, and this is can lead to overestimation of invasion. However, this is of doubtful clinical consequence as partial nephrectomy is rarely offered to unilateral WT.8

Evaluation of renal capsule is again difficult in the presence of large tumors, especially in children in whom the perinephric fat is also limited. The interobserver agreement was also only little better than fair in assessment, reiterating the difficulty. However, CT scan helps in ruling out invasion with NPV of 82 to $92 \%$ in our study.

Other studies have found similar difficulties in assessment of the capsule and lymph nodes with malignant abdominal tumors in general and with WT specifically. DamgaardPedersen et al suggest that capsular determination in children is difficult because of the sparse amount of perivisceral fat in tissue plane and nodal evaluation is difficult because of the paucity of fat in retroperitoneal tissue planes. ${ }^{10}$

Our study had certain limitation, with the most significant being that it was a retrospective study. It also did not have equal distribution of cases across the stages, with most cases being stage II. The lower incidence of stage I disease could be because we are a tertiary cancer center and hence more likely to treat advanced cases. The incidence of stage III is also low as we routinely administer preoperative chemotherapy to most of our patients.

\section{Conclusions}

CT is a valuable diagnostic tool for evaluating WT. The diagnostic accuracy of CT scan for predicting the likely stage improves with advanced stage of the disease, where surgery is more exigent. CT is extremely valuable in assessment of tumor thrombus and in ruling out invasion of adjacent organs and nodal disease. Therefore, imaging has the potential to optimize the therapeutic approach and may thus help in improving outcomes, especially in the resource-limited countries.

Table 7 Overall validity of CT scan (after concordance between two radiologists)

\begin{tabular}{|l|l|l|l|l|}
\hline Parameters & Sensitivity (\%) & Specificity (\%) & PPV (\%) & NPV (\%) \\
\hline PCS involvement & 79 & 50 & 45 & 82 \\
\hline Ureteric spread & 41.5 & 93 & 46.5 & 92 \\
\hline Adenopathy & 50 & 83 & 19 & 95 \\
\hline Capsular invasion & 84 & 67.5 & 62.5 & 87 \\
\hline Sinus involvement & 71.5 & 59.5 & 70.5 & 60 \\
\hline $\begin{array}{l}\text { Renal vein } \\
\text { thrombosis }\end{array}$ & 100 & 100 & 100 & 100 \\
\hline
\end{tabular}

Abbreviations: CT, computed tomography; NPV, negative predictive value; PCS, pelvicalyceal system; PPV, positive predictive value. 
The treatment strategy should be based on the wellestablished surgical and pathologic staging systems to avoid the hazards of inaccurate staging.

\section{Funding}

None.

\section{Conflict of Interest}

None declared.

\section{References}

1 Tongaonkar HB, Qureshi SS, Kurkure PA, Muckaden MA, Arora B, Yuvaraja TB. Wilms' tumor: an update. Indian J Urol 2007;23(4):458-466

2 Hörmann M, Puig S, Wandl-Vergesslich K, Prokesch R, Lechner G. [Radiologic staging of thoracic and abdominal tumors in childhood] [in German]. Radiologe 1999;39(7):538-545

3 Kaste SC, McCarville MB. Imaging pediatric abdominal tumors. Semin Roentgenol 2008;43(1):50-59
4 Kembhavi SA, Qureshi S, Vora T, et al. Understanding the principles in management of Wilms' tumour: can imaging assist in patient selection? Clin Radiol 2013;68(7):646-653

5 Gow KW, Roberts IF, Jamieson DH, Bray H, Magee JF, Murphy JJ. Local staging of Wilms' tumor-computerized tomography correlation with histological findings. J Pediatr Surg 2000;35(5):677-679

6 Abdelmaboud Ibrahim NM, Elsaid HH, Aly Aboubeih E. The accuracy of MDCT for staging of Wilms' tumor. Egyptian J Radiol Nucl Med 2014;45(2):575-581

7 Abdelhalim A, Helmy TE, Harraz AM, Abou-El-Ghar ME, Dawaba ME, Hafez AT. Can computerized tomography accurately stage childhood renal tumors? J Urol 2014;192(1):194-199

8 Metzger ML, Dome JS. Current therapy for Wilms' tumor. Oncologist 2005;10(10):815-826

9 Silva EJC, Silva JAP. Local behavior and lymph node metastases of Wilms' tumor: accuracy of computed tomography. Radiol Bras 2014;47(1):9-13

10 Damgaard-Pedersen K, Yssing M, Mauritzen K. CT in the staging of children with malignant tumours. Pediatr Radiol 1982;12(3):139-143 